\title{
Development and Implementation of Pediatric Nursing-Clinical Decision Support System for Hyperthermia: A Pre- and Post-Test
}

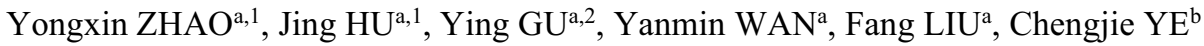 \\ and Xiaobo $\mathrm{ZHANG}^{\mathrm{c}, 2}$ \\ ${ }^{1}$ First authors, ${ }^{2}$ Corresponding authors \\ ${ }^{a}$ Nursing Department, Children's Hospital of Fudan University, \\ Shanghai, People's Republic of China \\ ' Information and Technology Department, Children's Hospital of Fudan University, \\ Shanghai, People's Republic of China \\ 'Dean's Office, Children's Hospital of Fudan University, \\ Shanghai, People's Republic of China
}

\begin{abstract}
Firstly, we form the Pediatric Nursing-Knowledge Base for Hyperthermia, which combines publicly clinical practice guidelines and nursing routines of hyperthermia management. Then, following the nursing process framework, the system is developed by clinical decision support technology. Finally, a pre-and posttest is adopted to examine the effectiveness, usability and feasibility before and after using the system. Its effectiveness is examined by nursing records quality including completeness of nursing assessment, timeliness of nursing diagnosis, individualization of nursing interventions, and timeliness of nursing evaluation. Its usability and feasibility are assessed using the Clinical Nursing Information System Effectiveness Evaluation Scale. There is a significant difference between the two groups in effectiveness, usability and feasibility. Although the system is developed specifically for our hospital workflow and processes, the Pediatric NursingKnowledge Base for Hyperthermia and workflow for hyperthermia management in this study can be used as a reference to other hospitals.
\end{abstract}

Keywords. Clinical decision support system, hyperthermia, nursing information, pediatric, knowledge base

\section{Introduction}

Fever is one of the most common presenting complaints of children admitted to the hospital. Prolonged fever will increase basal metabolic rate and oxygen consumption, which will affect many organ systems such as cardiopulmonary, digestive, and nervous systems. Nurses, as the healthcare professionals who interact most with children and parents during hospitalization. However, in clinical practice, the evidence or knowledge of hyperthermia nursing management is scarce. some nurses exhibited a corresponding

\footnotetext{
${ }^{1}$ Corresponding Author, Ying Gu and Xiaobo Zhang, No. 399, Wanyuan Road, Shanghai, China.
} 
lack of knowledge concerning fever, inconsistent treatment approaches along with fever phobia and a lack of knowledge were exhibited in certain studies. [1-3] Researches on the application of nursing process to fever were rarely presented, so the problems that nurses encountered in nursing process for fever were not clear. In our hospital, nurses focus on cooling mostly, and pay less attention to the comfort of children, psychological state and the behavior of caregivers in the nursing process of fever. Moreover, nursing assessment, diagnosis, intervention and evaluation were independent from each other and lacking restriction in our original nursing information system, nursing process for fever was easily interrupted.

A clinical decision support system (CDSS) is a knowledge-based information system that utilizes and integrates the characteristics of individual patients with a computerized knowledge base to generate patient-specific assessments and enhanced clinical decisions. [4] At present, there are some studies that apply CDSS to manage patients with fever in the emergency department and COVID-19 management. Few studies focus on hospitalized patients, let alone hospitalized children. Fever is a medical diagnosis, and its corresponding nursing diagnosis is hyperthermia. Based on the framework of nursing process, this study combined evidence-based nursing and clinical decision support technology to develop a new system called Pediatric Nursing - Clinical Decision Support System for Hyperthermia (PedN-CDSS- hyperthermia) to improve nursing practice and ensure the continuity and integrity in nursing care for hyperthermia.

\section{Methods}

This study consisted of two stages. The first stage was PaedN-CDSS- hyperthermia development, which was then continued by its preliminary examination of the implementation before (1-31 August 2018) and after (1-31 December 2019) using PaedN-CDSS-Hyperthermia.

\subsection{PaedN-CDSS-Hyperthermia Development}

PaedN-CDSS-Hyperthermia combined hyperthermia nursing management with CDSS. Since the beginning of its development, this system integrated all steps of the nursing process, the conduction of medical advice, and the nursing task list. The system was designed based on several ideas. First, it combined publicly clinical practice guidelines and nursing routines to form a complete and scientific pediatric nursing knowledge basehyperthermia (Paed-NKB- hyperthermia). Second, as the nursing process is similar to a journey, CDSS should be multi-functional along the way, from reminder, notification alert, decision-making tool, and information storage; all of which are used to control all aspects of hyperthermia nursing management to ensure standardized and safe nursing practice. Next, this system was interconnected to the Hospital Information System (HIS) to break any information barriers between doctors and nurses. Besides that, it was built to prescribe prn medical orders to ensure the timeliness of cooling. In addition, this application should be able to create nursing task-list by listing down the nursing to-do items and setting reminders/alerts to ensure timely nursing interventions. Finally, it could generate an automated and structured electronic nursing documents to achieve uniform and standardized nursing records.

This system adopted Browser/Server architecture, it's back-end used java language and front-end uses C\# language. it treated computer and personal digital assistant as 
hardware, wired and wireless LAN as networks, supporting the joint operation of the computer and Personal Digital Assistant (PDA). This system included 4 modules, including nursing assessment, nursing plan, prn medication orders, and nursing task list (Figure 1).

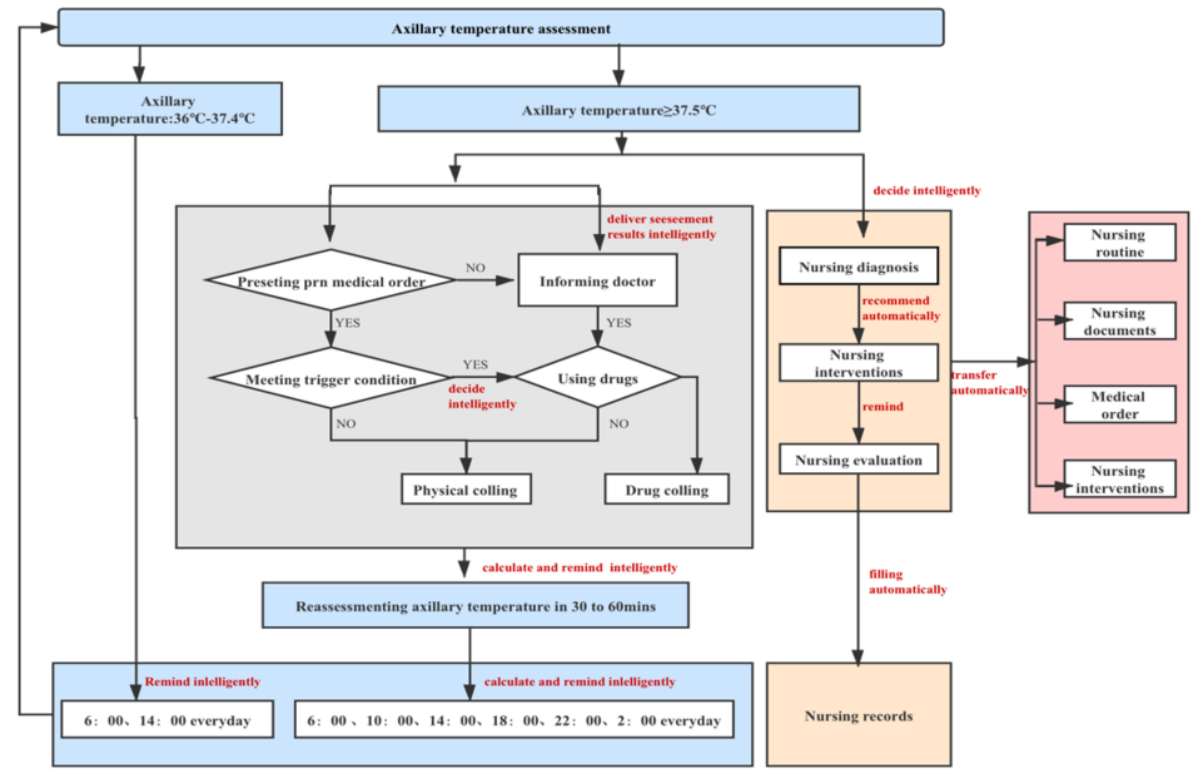

Figure 1. PedN-CCDSS-hyperthermia modules.

\subsection{PaedN-CDSS-Hyperthermia Implementation}

Before ( 1 to $31^{\text {st }}$ August 2018)and after (1 to 31 st December 2019)steady using PaedNCDSS-Hyperthermia, we used the Clinical Nursing Information System Effectiveness Evaluation Scale (CNISEES) [5] to test the system usability and feasibility by nurse's using experience. we used the nursing quality to examine its effectiveness. The quality of nursing records was measured by the completeness of nursing assessment, the timeliness of nursing diagnosis, the individualization of nursing interventions, and the timeliness of nursing evaluation. Specifically, Completeness of nursing assessment means actual evaluation times are equal to the theoretical evaluation times according the degree of fever and whether to take medication. Timeliness of nursing diagnosis means nursing diagnosis is made within 30 minutes of the first axillary temperature of $37.5{ }^{\circ} \mathrm{C}$ or higher. Individualization of nursing intervention means the contents of nursing intervention are consistent with the patient's condition and actual treatment methods, this index was evaluated back-to-back by the head nurse of the two study ward, when the results were in dispute, head nurse in another ward was invited to make a decision. Timeliness of nursing evaluation means nurse evaluates the nursing outcome within 72 to 86 hours of continuous normal axillary temperature, and children with normal axillary temperature less than 72 hours or still in fever but discharged from hospital is also considered to be timely. 


\section{Results}

The total score of CNISEES was $67.52 \pm 6.36$ vs. $93.88 \pm 3.71$ in control group and experimental group respectively $(\mathrm{t}=-33.073, \mathrm{P}=0.000)$. There was significant difference between the two groups in dimension of "information quality", "service quality", "customer satisfaction" and "net income" $(p<0.01)$, but there was no sig significant difference between the two groups in dimension of "system quality" $(\mathrm{p}=0.19)$. The results of specific items showed "response time", "safety", and "accuracy" between the two groups was no significant difference $(\mathrm{P}>0.05)$.

In terms of the completeness of nursing assessment, 32 nursing records were incomplete among controls after taking cooling measures but no incomplete assessment in the experimental group, $\left(\chi^{2}=40.46, \mathrm{p}<0.001\right)$. In terms of the timeliness of nursing diagnosis, 12 nursing records were delayed for 3 days or more with the longest delay period was for 14.52 days among controls, In the experimental group, 7 nursing records were delayed up to 13.55 hours $(\chi 2=51.60, \mathrm{p}<0.001)$. In terms of the timeliness of nursing evaluation, 62 nursing records were delayed with the maximum delaying of 14 days in the control group, but no delay was observed in the experimental group $(x$ $2=97.67, \mathrm{p}<0.001)$.

Moreover, before using PedN-CDSS-Hyperthermia, there were two terminologies used in the nursing diagnosis for "axillary temperature $\geq 37.5^{\circ} \mathrm{C}$ ": "hyperthermia" and "fever". The nursing interventions were also already fixed and cannot be changed, with differences only in the "wards" or "nursing diagnosis". After using the PedN-CDSSHyperthermia, there was only one nursing diagnosis, "hyperthermia", and the nursing interventions could vary from child to child.

\section{Discussion}

As the change of information system requires a transitional period generally lasting for 12 to 18 months to adapt to and learn, if there are problems with equipment or software, nurses may have negative experiences during this period. [6] PedN-CDSS Hyperthermia is still in transition period now, several technical problems had occurred, such as system obstruction, data loss, and blank screen of computer or PDA, which led to the impression of the system's underperformance among the nurses. However, it is important to note that the original nursing information system had been used in this hospital for many years, and nurses had been accustomed to its mode and function, so the "system quality" of the new system had not been significantly improved. The nursing process for patients requires the nurses to be able to obtain the best information, think about the information, filter the information, and make clinical decision. [7] High satisfaction related to a number of factors, such as usability, reliability, and support provided when problems occurred. The PedN -CDSS-Hyperthermia was able to trigger nursing diagnosis, recommend nursing interventions, remind nursing evaluation, estimate the reassessment time point, fill out the structured nursing documents, and inform the doctor automatically, which provides reliable care information and ensures the quality of collected data. In the process of building the PedN -CDSS-Hyperthermia, members of multi-disciplinary team communicated with the clinical nurses timely through the WeChat or face-to-face to solve problems encountered in system operation, which was the main reason for a better experience to "service quality" feature. 
As PedN-CDSS-Hyperthermia could automatically extract each step of the nursing process and fill them into the standardized, structured, and formatted nursing record sheet, which could prevent missing contents in the records effectively and improve the quality of nursing records.

Because the system was specifically developed according to our hospital's hyperthermia management workflow and procedures, its generalizability may be limited to other hospital. However, the workflow is based on nursing process, and the knowledge base is based on evidence-based nursing for hyperthermia. We believe that the knowledge base and workflow for hyperthermia in this study can be used as a reference to other hospitals.

\section{Conclusion}

Based on the framework of nursing process, PedN-CDSS-Hyperthermia combined evidence-based nursing and clinical decision support technology, which included nursing assessment, nursing plan, medical order and nursing task list 4 modules. PedN-CDSSHyperthermia generally enhanced the nursing records quality and nurse's system use experience, standardized the hyperthermia nursing process and improved the timeliness, continuity and integrity of hyperthermia care for hospitalized children.

\section{Acknowledgements}

The authors thank all the participants who are contributed to the study, and thank the nursing department, IT department, the QC department, and Ewell technology company supporting this study.

\section{References}

[1] Urbane UN, Likopa Z, Gardovska D et al. Beliefs, practices and health care seeking behavior of parents regarding fever in children. Medicina ,2019,55(7):398.

[2] Davis T. NICE guideline: Feverish illness in children-Assessment and initial management in children younger than 5 years. Arch. Dis. Child. Educ. Pract,2013, 98, 232-35.

[3] Hiller MG, Caffery MS, Bégué RE. A survey about fever knowledge, attitudes, and practices among parents. Clin Pediatr (Phila), 2019 ,58(6):677-80.

[4] Ortiz DR, Maia FO, Ortiz DC et al. Computerized clinical decision support system utilization in nursing: A scoping review protocol. JBI Database of Systematic Reviews and Implementation Reports, 2017, 15(11):2638-44.

[5] Zhao YX, Gu Y, Zhang XB et al. Developed the clinical nursing information system effectiveness evaluation scale based on the new D\&M model and conducted reliability and validity evaluation. Chin J Prac Nurs, 2020,36(7):50-56.

[6] Huang C, Zhan YY, Liu XY et al. The application progress of mobile medical in nursing field. Chin J Nurs, 2019,54(8):1264-9.

[7] Hao AT, Hsu CY, Li FH et al. Apply creative thinking of decision support in electrical nursing record. Stud Health Technol Inform,2006,124:313-9. 